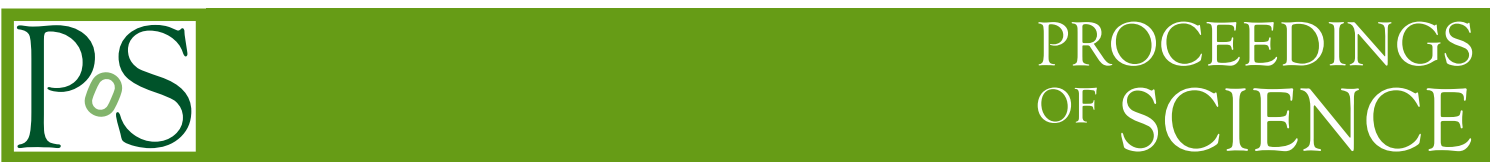

\title{
Measurements of Atmospheric Neutrinos using the MINOS Detector
}

\author{
Luke Corwin*ث \\ Indiana University \\ E-mail: lacorwin@indiana.edu
}

\begin{abstract}
This poster presents the latest atmospheric neutrino results from the MINOS experiment. The results are based on a data set of 1657 live-days and combine together observations of contained vertex neutrino interactions and neutrino-induced upward muons in the MINOS far detector. The measured curvature of muons in the MINOS magnetic field is used to separate neutrinos and antineutrinos, and the observed ratio of neutrinos to anti-neutrinos is compared to the Monte Carlo (MC) expectation. The data are separated into bins of L/E resolution, and a maximum likelihood fit to the observed $\mathrm{L} / \mathrm{E}$ distributions is used to determine the oscillation parameters separately for neutrinos and anti-neutrinos. Confidence limits are placed on the difference between these oscillation parameters. The techniques and current status of an analysis using this data set to search for the neutrino mass hierarchy are also reported.
\end{abstract}

35th International Conference of High Energy Physics

July 22-28, 2010

Paris, France

*Speaker.

${ }^{\dagger}$ For the MINOS Collaboration. 
Cosmic rays impinging on the atmosphere produce cascades of particles, including neutrinos. Oscillation of $v_{\mu}$ to other flavors appears as a $v_{\mu}$ deficit for certain values of path length (L) and $v$ energy (E), where $\mathrm{L}$ varies from $\sim 10$ to $\sim 13000 \mathrm{~km}$. MINOS measures L/E across 4 orders of magnitude. Oscillations in $v_{\mu}$ and $\bar{v}_{\mu}$ are detected. Between 1 Aug. 2003 and 1 Aug. 2008, 1657 live days (24.6 kton-Yrs) of data were collected at the MINOS far detector [2].

We use a high resolution subset of data with clean tracks and well-measured charge to calculate the ratio of $v_{\mu}$ to $\bar{v}_{\mu}$ events. We compare this ratio for data and MC (using MINOS beam oscillation parameters [1]) to check the consistency of $v_{\mu}$ with $\bar{v}_{\mu}$ oscillations. The resulting double ratio, $\frac{R_{v / V}^{\text {data }}}{R_{v / V}^{\mathrm{MC}}}=1.04_{-0.10}^{+0.11} \pm 0.10$, is consistent with unity and thus with equal oscillations for $v_{\mu}$ and $\bar{v}_{\mu}$.

We divide the data into bins of $\mathrm{L} / \mathrm{E}$ resolution. In the limit of two flavor oscillations $\left(v_{\mu} \leftrightarrow v_{\tau}\right)$, we perform a maximum likelihood fit to the reconstructed L/E distributions to obtain oscillation parameters. For our $v$ sample, we fix the $\bar{v}$ parameters to the MINOS $v$ best fit oscillation parameters from 2008 [1]. Then, we perform the opposite analysis for our $\bar{v}$ sample. Systematic uncertainties are fitted as nuisance parameters. Results are shown in Figure 1. In Equations 1-3, limits and uncertainties are at the $90 \%$ confidence level.

$$
\begin{gathered}
\left|\Delta m_{32}^{2}\right|=2.6_{-1.3}^{+4.4} \times 10^{-3} \mathrm{eV}^{2} \\
\left|\Delta \bar{m}_{32}^{2}\right|=1.8_{-0.6}^{+1.5} \times 10^{-3} \mathrm{eV}^{2}, 7 \times 10^{-3}<\frac{\left|\Delta \bar{m}_{32}^{2}\right|}{\mathrm{eV}^{2}}<17 \times 10^{-3} \\
\sin ^{2}\left(2 \theta_{23}\right)>0.58, \sin ^{2}\left(2 \bar{\theta}_{23}\right)>0.60
\end{gathered}
$$

To test the consistency of the $v$ and $\bar{v}$ mass differences, we perform our fit with the mixing angles fixed to maximal mixing and the mass differences allowed to vary. The results are consistent within uncertainties:

$$
\left|\Delta m_{32}^{2}\right|-\left|\Delta \bar{m}_{32}^{2}\right|=0.4_{-1.2}^{+2.5} \times 10^{-3} \mathrm{eV}^{2}
$$

The ability of MINOS to distinguish between $v$ and $\bar{v}$ allows us to search for the mass hierarchy. The number of $\mu^{+}$and $\mu^{-}$, for certain values of energy and angle, depends on the mass hierarchy. We are cur-
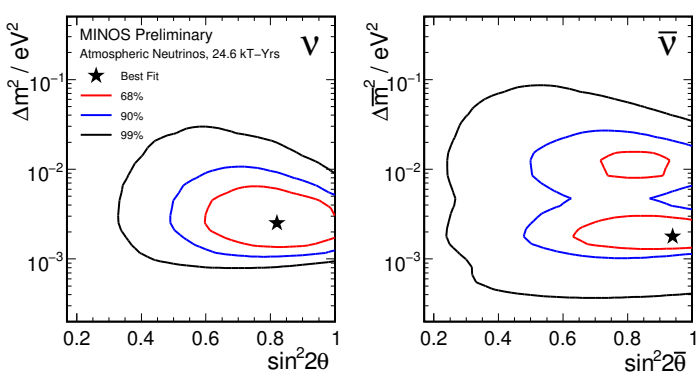

Figure 1: Confidence level contours given by the atmospheric neutrino analysis on MINOS.

rently determining how to use statistical tests (such as $\chi^{2}$ or Kolmogorov-Smirnov) to compare the atmospheric data with simulations for the two hierarchies and calculate which hierarchy is more consistent with our data. We plan to include the additional data taken since 1 Aug. 2008 in this search.

\section{References}

[1] P. Adamson et al. Phys. Rev. Lett. 101, 131802 (2008) [arXiv:0806.2237 [hep-ex]].

[2] D. G. Michael et al. Nucl. Instrum. Meth. A 596, 190 (2008) [arXiv:0805.3170 [physics.ins-det]]. 